\title{
Evaluación diagnóstica de habilidades de pensamiento e informacionales a través del diseño y aplicación de tres instrumentos para estudiantes de primer grado de educación primaria
}

\author{
Diagnostic Evaluation of Thinking and Information Skills Through the Design and \\ Application of Three Instruments for Children at First Grade of Elementary School

\section{Avaliação diagnóstica de habilidades de pensamento e informação através do desenho e aplicação de três instrumentos para crianças na primeira série do ensino fundamental}

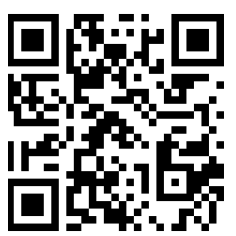

Mariela González-López

Universidad Autónoma de Chihuahua

Chihuahua, México

marieladeangel@hotmail.com

http://orcid.org/0000-0002-3178-8000

Juan Machin-Mastromatteo

Universidad Autónoma de Chihuahua

Chihuahua, México

jmachin@uach.mx

http://orcid.org/0000-0003-4884-0474

Javier Tarango

Universidad Autónoma de Chihuahua

Chihuahua, México

jtarango@uach.mx

D http://orcid.org/0000-0002-0416-3400

Recibido • Received • Recebido: 10 / 01 / 2019

Corregido • Revised • Revisado: 26 / 06 / 2020

Aceptado • Accepted • Aprovado: 18 / 08 / 2020

\begin{abstract}
Resumen: Este artículo toma como referencia las problemáticas de la falta de habilidades para la lectura, las matemáticas y la ciencia, según la realidad del sistema educativo público mexicano, así como los resultados obtenidos en las evaluaciones promovidas por la Organización para la Cooperación y el Desarrollo Económico (OCDE). La investigación se basó en un estudio cuantitativo, el cual se obtuvo de una evaluación diagnóstica sobre el desarrollo de habilidades informacionales y de pensamiento en la niñez de educación básica, para lo cual se diseñaron y se aplicaron tres instrumentos sobre habilidades de pensamiento, pensamiento matemático, así como competencias informacionales, de lenguaje y de comunicación en la niñez, para conocer el nivel de habilidades desarrolladas previo a incorporarse al programa del nuevo modelo educativo propuesto por la Secretaría de Educación Pública (SEP) de México. Se aplicó el método diagnóstico de situación educativa (DS): i) un examen de 20 ejercicios para medir el nivel de desarrollo de cada habilidad en estudio; ii) una rúbrica para evaluar las respuestas de la niñez; y iii) una tabla para anotar los resultados de cada habilidad. Las conclusiones de esta evaluación diagnóstica incluyen que este estudiantado, que se encuentran
\end{abstract}


http://doi.org/10.15359/ree.24-3.21

http://www.una.ac.cr/educare

educare@una.ac.cr

en la etapa preoperatoria, tiene cierto desarrollo en sus habilidades de pensamiento (observación, comparación, clasificación, ordenación, inferencias, memoria y narración de historias); habilidades lingüísticas (escriben su nombre sin apellidos, comunican emociones, narran historias breves sobresale el lenguaje oral); y habilidades informacionales (no se tienen nociones de conceptos que intervienen).

Palabras claves: Evaluación; alfabetización informacional; habilidades de pensamiento; lenguaje; educación primaria.

Abstract: This article takes as reference the problems that exist due to the lack of reading, mathematics and science skills, according to the reality of the Mexican public education system, as well as the results obtained in the evaluations promoted by the Organisation for Economic Cooperation and Development (OECD). The research was based on a quantitative study, which was conducted from a diagnostic evaluation on the development of information and thinking skills in children in basic education school. This research designed and applied three instruments on thinking skills, mathematical thinking, and information, language and communication skills in children. The research aimed to know the level of skills developed prior to joining the program of the new educational model proposed by the Ministry of Public Education (SEP) of Mexico when applying the method of the diagnosis of educational situation (DS) that has the following components: i) an examination of 20 exercises to measure the level of development of each skill under study; ii) a rubric to evaluate the children's responses; and iii) a table to record the results of each skill. The diagnostic evaluation concluded that students, who are in the preoperative stage, have some development in their thinking skills (observation, comparison, classification, ordering, inferences, memory and storytelling), linguistic skills (they write their name, without surnames, communicate emotions, tell short stories, oral language stands out), and informative skills (there are no notions of concepts that intervene).

Keywords: Evaluation; information literacy; thinking skills; language; elementary education.

Resumo: Este artigo toma como referência as problemáticas da falta de habilidades para a leitura, a matemática e a ciência, de acordo com a realidade do sistema de ensino público mexicano, bem como os resultados obtidos nas avaliações promovidas pela Organização para Cooperação e Desenvolvimento Econômico (OCDE). A pesquisa foi baseada em um estudo quantitativo, que foi obtido a partir de uma avaliação diagnóstica sobre o desenvolvimento de habilidades de informação e pensamento em crianças da educação fundamental, para as quais foram protejados e aplicados três instrumentos sobre habilidades de raciocínio, pensamento matemático e habilidades de informação, linguagem e comunicação em crianças, para conhecer o nível de habilidades desenvolvidas antes de aderir ao programa do novo modelo educacional proposto pela Secretaria da Educação Pública (SEP) do México. Foi aplicado o método diagnóstico de Situação Educacional (DS): i) um exame de 20 exercícios para medir o nível de desenvolvimento de cada habilidade em estudo; ii) uma rubrica para avaliar as respostas das crianças; e iii) uma tabela para registrar os resultados de cada habilidade. As conclusões dessa avaliação diagnóstica incluem que estes estudantes que se encontram na etapa pré-operatória, possuem algum desenvolvimento em suas habilidades de pensamento (observação, comparação, classificação, ordenação, inferências, memória e narrar histórias); habilidades linguísticas (escrevem seu nome, sem sobrenomes, comunicam emoções, narram histórias curtas, destacam-se na linguagem oral); e habilidades informativas (não possuem noções de conceitos que intervêm).

Palavras chave: Avaliação; alfabetização informacional; habilidades de pensamento; linguagem; educação fundamental. 


\section{Introducción}

La importancia de una buena planeación en el ámbito escolar debe ser un punto esencial y prioritario. Para ello, debe usarse la intervención pedagógica como práctica educativa y dispositivo para: recabar información escolar, acercarse a la realidad del grupo de estudiantes y la contribución en el éxito del proceso de enseñanza aprendizaje, por ello se debe realizar un diagnóstico para el éxito de la planeación (Arriaga Hernández, 2015; OCDE, 2011).

Reafirmando la importancia de los diagnósticos, Lovatón Sarco (2012) señala que, en particular, el diagnóstico de situación educativa (DS) o evaluación diagnóstica se refiere a una herramienta fundamental en la elaboración del currículo para la formación académica, el cual puede conformarse de ejercicios, los cuales brindan información previa de las capacidades que tiene desarrolladas el estudiantado o de los conocimientos de algún tema, según las actividades propuestas. Tales perspectivas son complementadas por Meléndez y Gómez (2008) y Moreno Olivos (2012), quienes proponen una planificación curricular con secuencia lógica e interdisciplinaria, en donde, además de la fase diagnóstica, se identifiquen los propósitos, estrategias, metodologías, herramientas y formas de evaluación, considerando así, la planificación de la enseñanza basada en competencias.

De acuerdo con la perspectiva de la Organisation for Economic Co-operation and Development [OECD] (2017a), el estudiantado que en una evaluación diagnóstica muestra mayores habilidades en conocimientos científicos, lectura y matemáticas, tiende a poseer mayores capacidades en la solución de problemas. La gestión de información, así como los procesos de gestión de datos, permiten desarrollar habilidades de razonamiento. Además, la misma OECD (2017b) afirma que todo esto se refleja en su medición, por ejemplo, a través del examen PISA, el cual se centra en la ciencia, lectura, matemáticas y la resolución de problemas colaborativos, así como de enfoque financiero del sujeto evaluado.

En la docencia, es común que se desarrollen DS, especialmente cuando no se puede realizar algo o actuar sobre una cosa, si esta no se conoce. Alternativamente, esta evaluación también se realiza para guiar la planeación a futuro de un proceso de enseñanza-aprendizaje, que sea acorde con el grado educativo en cuestión (Hidalgo y Murillo, 2017; Morán, 2004). El DS sirve para que el personal docente o investigador obtenga una concepción sobre conocimientos previos, como aquellos correspondientes al manejo de la lectoescritura, además de permitirles mejorar su práctica docente y el proceso de aprendizaje de sus estudiantes. Esto justifica la importancia del DS en el quehacer docente, en la planificación de los programas de estudios y en las políticas pedagógicas (Barboza Marcano, 2007). Los resultados de un DS guían al personal docente a programar actividades que le permitan conducir un aprendizaje enfocado en la adquisición de conocimientos, habilidades, actitudes, valores y competencias para el desarrollo integral del estudiantado. Este objetivo es acorde con los aprendizajes esperados en los programas de estudio de educación primaria en México (Secretaría de Educación Pública [SEP], 2017b). 
http://doi.org/10.15359/ree.24-3.21

http://www.una.ac.cr/educare

educare@una.ac.cr

Dado que la actividad diagnóstica permite al cuerpo docente mejorar las prácticas educativas en las áreas de trabajo de la docencia, este artículo plantea como objetivo principal el diseño y aplicación de un procedimiento para trabajar con estudiantes de primer grado de educación básica, de entre cinco y seis años de edad. Este hace posible comprender las problemáticas que se suscitan en la realidad cotidiana del aula, a través de la obtención de saberes necesarios, por medio de actividades para el desarrollo de procesos cognitivos, en cuanto a su nivel de desarrollo en las habilidades básicas de pensamiento, pensamiento matemático, así como competencias informacionales, lenguaje y comunicación. El diseño de esta investigación es de tipo cualitativo y realizado a través de la metodología de un diagnóstico de situación educativa, la cual busca diseñar estrategias para desarrollar tales habilidades.

\section{Estudios relacionados con el desarrollo de habilidades del pensamiento e informacionales}

Los estudios sobre el desarrollo de habilidades del pensamiento han sido de fuerte influencia en el desarrollo de procesos educativos, especialmente en la educación básica. Algunos estudios han llegado al nivel de validación de instrumentos para evaluar procesos cognitivos básicos y superiores, mediante la comprensión lectora, tomando en cuenta los dominios cognitivos y lingüísticos (González-López, 2019; 2020; Tapia y Luna, 2010). Otras investigaciones basan su propuesta en corrientes educativas como el paradigma constructivista de la enseñanza y aprendizaje en el desarrollo de pensamiento crítico y creativo (Muria Vila y Díaz Milagros, 2008). Ambas vertientes coinciden en los elementos para medir la ejecución (analizar, criticar y juzgar), la creatividad (descubrir, inventar e imaginar) y la practicidad (aplicar, usar y utilizar).

Los estudios de esta naturaleza toman mayor reconocimiento cuando se sostiene que hacer posible que las personas sigan aprendiendo durante todo el desarrollo personal implica que, en la etapa del nivel básico, se haya enseñado a aprender a aprender (Rodríguez, 2008). Por tanto, se forman sujetos con habilidades de pensamiento, de quienes se espera, ante tales procesos formativos en edad temprana, logren posteriormente evolucionar hacia el logro de una capacidad para identificar vínculos con procesos de investigación (Araya, 2014; Bahmani, 2016).

Aunado a lo anterior, las demandas sociales actuales proponen que la totalidad de estudiantes, sin importar el nivel educativo que curse, deberá adquirir, además de habilidades de pensamiento, habilidades informacionales, con lo cual se adquiere una formación integral, caracterizada por la capacidad de búsqueda de información y con énfasis en resultados de aprendizaje específicos (Heichman, 2008; Morales et al., 2018). Como resultado de esta caracterización educativa moderna, se espera que el sujeto sea capaz de aprender contenidos a nivel más profundo y mejorar su capacidad para comunicar ideas, e influir en la capacidad para solucionar problemas y para el aprendizaje permanente (Paul y Elder, 2003).

De forma individual, el desarrollo de habilidades informacionales, especialmente en escuelas de educación básica, garantiza las obligaciones y los derechos básicos del estudiantado en cuanto a su educación integral (Zubac y Tufekčić, 2014). A partir de esto surgen algunos intentos por 
http://doi.org/10.15359/ree.24-3.21

incorporar elementos relacionados con las habilidades informacionales, tales como: creación de juegos digitales para el aprendizaje para niños y niñas de 9 a 14 años (Costa et al., 2018); modelos para la resolución de problemas en la educación en escuelas de nivel básico, con énfasis en la efectividad y la validez para el aprendizaje constructivo (Joo y Park, 2015); generación de instrumentos para desarrollar y evaluar habilidades informacionales con miras a evitar la vulnerabilidad de la niñez ante sitios Web (Foo et al., 2014); evitar malas selecciones de contenidos y habilidades de evaluación de la información (Beck, 2015) y métodos para la enseñanza de habilidades informacionales por medio del dibujo (Brier y Lebbin, 2015), por mencionar los principales.

El desarrollo de las habilidades informacionales es un paradigma teórico-conceptual y metodológico que ha ido cambiando, en la última década, aunque mantiene su estado de inmadurez, especialmente en entornos educativos específicos, como es el caso de la educación básica (Ponjuan et al., 2015). Por eso el desarrollo de habilidades de pensamiento e informacional se tornan importantes en la educación infantil, ya que en las edades tempranas se desconocen los peligros detrás de la tecnología, así como su valor (Heider y Jalongo, 2015); igualmente, preocupa la preparación de docentes para enfrentar tales situaciones.

En un afán de justificar la importancia del desarrollo de habilidades informacionales en los niveles educativos básicos, se proponen las siguientes visiones: i) trabajar este tema más allá de la mera escritura y aritmética, estudiando prácticas que trasciendan los espacios formales de aprendizaje (Drotner y Kobbernagel, 2014); ii) durante el período de la infancia es más probable adquirir habilidades, tanto de pensamiento como informacionales, ya que el niño y la niña se encuentran en un periodo de desarrollo natural y cognitivo para adquirir experiencias transformadoras (Durand et al., 2005; Rodney-Wellington, 2014): y iii) resolver problemas suscitados por la sobreabundancia de información (Tamayo-Rueda et al., 2012). Por tanto, las habilidades informacionales deberán ser consideradas dentro de las políticas educativas.

\section{Planteamiento del problema}

El mundo actual muestra interconexión en múltiples aspectos como habilidades informacionales, digitales, de pensamiento, sociales, emocionales, ecológicas, flexibilidad cognitiva, creatividad, innovación, resolución de problemas y pensamiento crítico, por tanto, demanda individuos con rasgos globalizados del mundo contemporáneo (Blummer y Kenton, 2014; Navarro Navarro, 2018; Siu-Cheung, 2015). La educación básica no es la excepción, en donde se exige integrar programas y estrategias para desarrollar habilidades comunicativas, y amplia capacidad para resolver problemas. Es necesario, entonces, acrecentar en el estudiantado las habilidades de pensamiento, su capacidad lingüística y la competencia informacional (Amestoy de Sánchez, 2002; González-López et al., 2019; Zavala, 2014). Estas características deberían ser herramientas para el aprendizaje continuo en todas las etapas de la vida, situación que regularmente no sucede, especialmente en los niveles de bachillerato e incluso en la educación superior (Pompa Montes de Oca y Pérez López, 2015). 
http://doi.org/10.15359/ree.24-3.21

http://www.una.ac.cr/educare

educare@una.ac.cr

Una de las formas más conocidas como referente global de la condición educativa que observa cada país participante en cuanto a conocimientos, actitudes y habilidades en materia de lectura, matemáticas y ciencias es la prueba PISA, realizada por la OECD (2017b). Aunque los resultados de PISA ofrecen un análisis macrosocial en estudiantes de tercer grado de secundaria, es importante destacar que, en el caso de México, los resultados no han sido favorables, ya que la puntuación media es de 500 puntos, en lectura fue ligeramente mayor que en matemáticas; pero esta puntuación está por debajo de la media obtenida por otros países de la OCDE.

Tales resultados podrían atribuirse a problemas relacionados con los procesos de aprendizaje en la educación básica, en específico, en cuanto a la implantación de estrategias educativas. Desafortunadamente, las consecuencias de las debilidades educativas se hacen, de manera progresiva, más fuertes, por ejemplo, cuando el estudiantado muestra problemas reales de adaptación en otros niveles educativos superiores e incluso en el mercado laboral. Tales situaciones se presentan, en general, en estudiantes de países latinoamericanos, cuando participan en cualquier prueba estandarizada relacionada con la medición de habilidades informacionales. Por ejemplo, se ha comprobado tal condición al observar resultados en eventos en los que se han aplicado, por ejemplo, en las pruebas Standardized Assessment of Information Literacy Skills (SAILS) y la descontinuada iSkills (Lau et al., 2016).

Por ende, es necesario diseñar un diagnóstico para saber qué habilidades sobresalen en la niñez y cuáles no, para enseñar y practicar dichas habilidades para la vida, como son las habilidades de pensamiento, informacionales y lingüísticas. También es imperativo el desarrollo de competencias relacionadas con los procesos de comprensión y expresión, esto desde la perspectiva del aprendizaje, pues son fundamentales para el incremento cognitivo del estudiantado.

El desarrollo de estas competencias debe observar lo siguiente: suceder desde la educación básica y de forma transversal en todas las materias que integran el currículo. Específicamente en México, la SEP (2017b) indica la posibilidad de formar estudiantado libre y creativo, además con otras características, tales como: la crítica y la reflexión, elementos clave para mostrar capacidad en la solución de problemas planteados, tanto en ambientes académicos como de la vida misma. Por tanto, es necesario conocer de inicio, aquellas habilidades que posee el estudiantado antes de abordar los temas que constituyen los programas de estudio de primer grado.

\section{Metodología}

La técnica utilizada para la metodología fue el DS, para el análisis de alternativas y la valoración de sus consecuencias (Vilar, 1992). Por lo tanto, en esta investigación solo se obtienen del diagnóstico datos relacionados con las diferentes habilidades que posee el estudiantado al inicio del ciclo escolar de primer grado de primaria. Además, permite determinar las necesidades y oportunidades del estudiantado, particularmente de la niñez de educación primaria entre las edades de seis y siete años en la ciudad de Chihuahua, México.

6 
http://doi.org/10.15359/ree.24-3.21

La investigación desarrollada fue de tipo exploratoria y descriptiva. El diagnóstico aplicado a la educación persiguió impactos positivos en el desarrollo de prácticas educativas contextualizadas que permitan mejorar la relación entre teoría y práctica. El DS se llevó a cabo para apoyar las necesidades educativas y así realizar una planeación didáctica de calidad en las intervenciones en primer grado de primaria; es fundamental en tal nivel, ya que los niños y las niñas están en la etapa de aprendizaje de la lectoescritura. En consecuencia, el DS permite identificar las habilidades desarrolladas en el estudiantado, plantear soluciones y diseñar actividades más precisas para la enseñanza de los contenidos relacionados con la lectoescritura. La metodología para llevar a cabo la investigación integró cinco fases principales:

a) Diseño del instrumento de la rúbrica para la evaluación del examen de diagnóstico situacional, integrado con una escala de evaluación (regular $[R]$ ), bien $[B]$ y muy bien $[\mathrm{MB}]$. Dicho instrumento fue aplicado en un salón de primer grado de primeria y su constó de habilidades de pensamiento, lingüísticas e informacionales, ya que la SEP (2017a, 2017b) señala la importancia de abordar un enfoque competencial para la enseñanza de los contenidos de cada grado escolar. Particularmente en el desarrollo de sus competencias, de manera tal que se logre una convergencia entre lo individual y lo social en aspectos ligados a lo cognoscitivo, afectivo y psicológico, que potencien una capacidad adaptativa al entorno (García Retana, 2011). Todo lo anterior tiene el fin de enfocar al estudiantado en el desarrollo de habilidades y hacia una formación para ser social, intelectual, ecológico y emocional; lo cual significa apostar en la educación de la niñez para una sociedad contemporánea.

b) La planeación y el diseño del segundo instrumento incluyó desarrollar ejercicios sobre las habilidades de pensamiento, informacionales, lenguaje y comunicación, los cuales fueron evaluados con la escala anteriormente mencionada. Dichas habilidades son claves para el desarrollo integral de la niñez.

c) En la intervención educativa se aplicó el examen a un grupo de primer grado de primaria, constituido por 27 participantes, entre ellos 14 niños y 13 niñas. Dicho examen integró 20 ejercicios sobre procesos cognitivos y habilidades informacionales, los cuales permitieron dar cuenta sobre las capacidades de cada estudiante, para las futuras orientaciones o planeaciones pedagógicas que guíen hacia una enseñanza enfocada en el desarrollo de competencias.

d) La muestra del estudiantado fue intencional, porque era el grupo con una mayor cantidad de estudiantes de primer grado en una escuela primaria específica, la cual, en general, cuenta con alrededor de 315 estudiantes en total y dos grupos más de primer grado de menor cantidad de niños y niñas. 
http://doi.org/10.15359/ree.24-3.21

http://www.una.ac.cr/educare

educare@una.ac.cr

e) Los tres instrumentos diseñados fueron validados por un panel experto, tanto en educación como en alfabetización informacional, que validó con los siguientes elementos aceptables: contenido, objetividad, observación, conceptualización, actualidad, intencionalidad, en aspectos de cantidad, calidad, pertinencia, escala, presentación y formalidad del instrumento.

\section{Instrumentos diseñados}

A continuación, se describen los tres instrumentos diseñados para su posterior aplicación.

\section{Primer instrumento: Examen diagnóstico}

Este instrumento fue utilizado para precisar el nivel de desarrollo de las veinte habilidades en estudio. Dichas habilidades incluyeron:

a) Habilidades de pensamiento matemático: observación, clasificación, interpretación, representación, inferencias, ordenación, comparación, evaluación, toma de decisiones, y memoria.

b) Habilidades de lenguaje y comunicación: escribir su nombre, narrar una historia, percepción, comunicación de emociones y autoevaluación.

c) Habilidades informacionales: define una tarea a realizar, estrategia para buscar información, localización y acceso a la información, análisis de información, síntesis de la información y evaluación de la información.

Es importante mencionar que, dada la edad de las personas participantes en el estudio, este cuestionario es principalmente visual, por lo tanto, se leyeron las instrucciones, se diseñaron imágenes para elaborar el examen, además de los ejercicios que pedían al estudiantado que realizaran un dibujo, para asegurar una mayor comprensión por parte de los niños y niñas, en cuanto a lo que requería cada ejercicio.

La Tabla 1 muestra el examen diagnóstico para evaluar habilidades del pensamiento e informacionales, donde la primera columna enumera la sección según la habilidad a evaluar; la segunda columna presenta la instrucción dada al estudiantado participante en cada ejercicio, junto a una breve descripción de la estrategia empleada, la cual se agrega solamente con el propósito de hacer la metodología más clara y precisa en este artículo; y la tercera columna muestra el dibujo usado en cada ejercicio, excepto para los ejercicios 10 y del 14 al 20, los cuales no usaron dibujos preparados para sus respectivas actividades. Este examen fue implementado al grupo elegido, leído y contestado por cada participante. 
http://doi.org/10.15359/ree.24-3.21

Tabla 1: Examen diagnóstico para evaluar habilidades del pensamiento, pensamiento matemático, informacionales, de lenguaje y comunicación

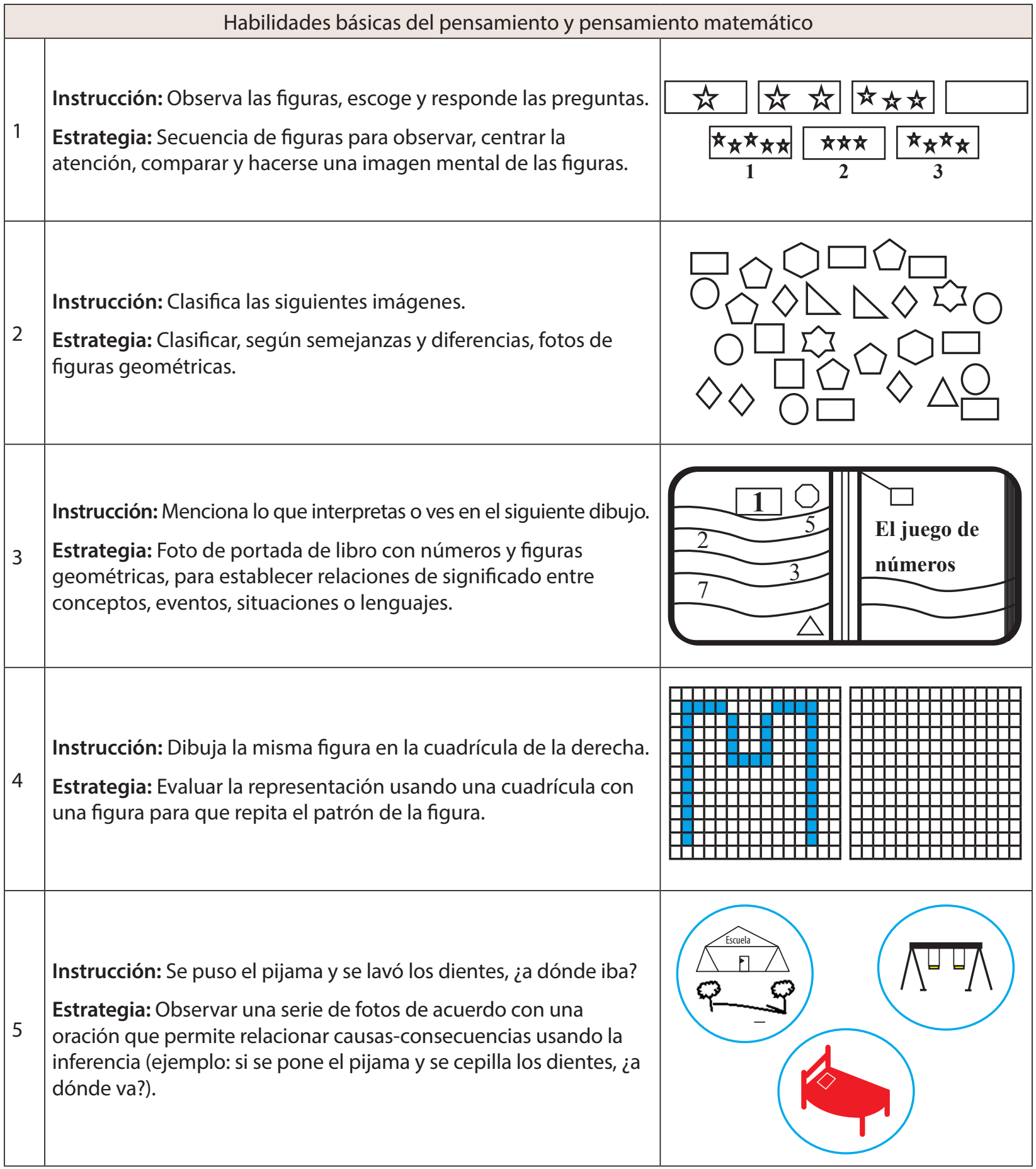

continúa 
http://doi.org/10.15359/ree.24-3.21

http://www.una.ac.cr/educare

educare@una.ac.cr

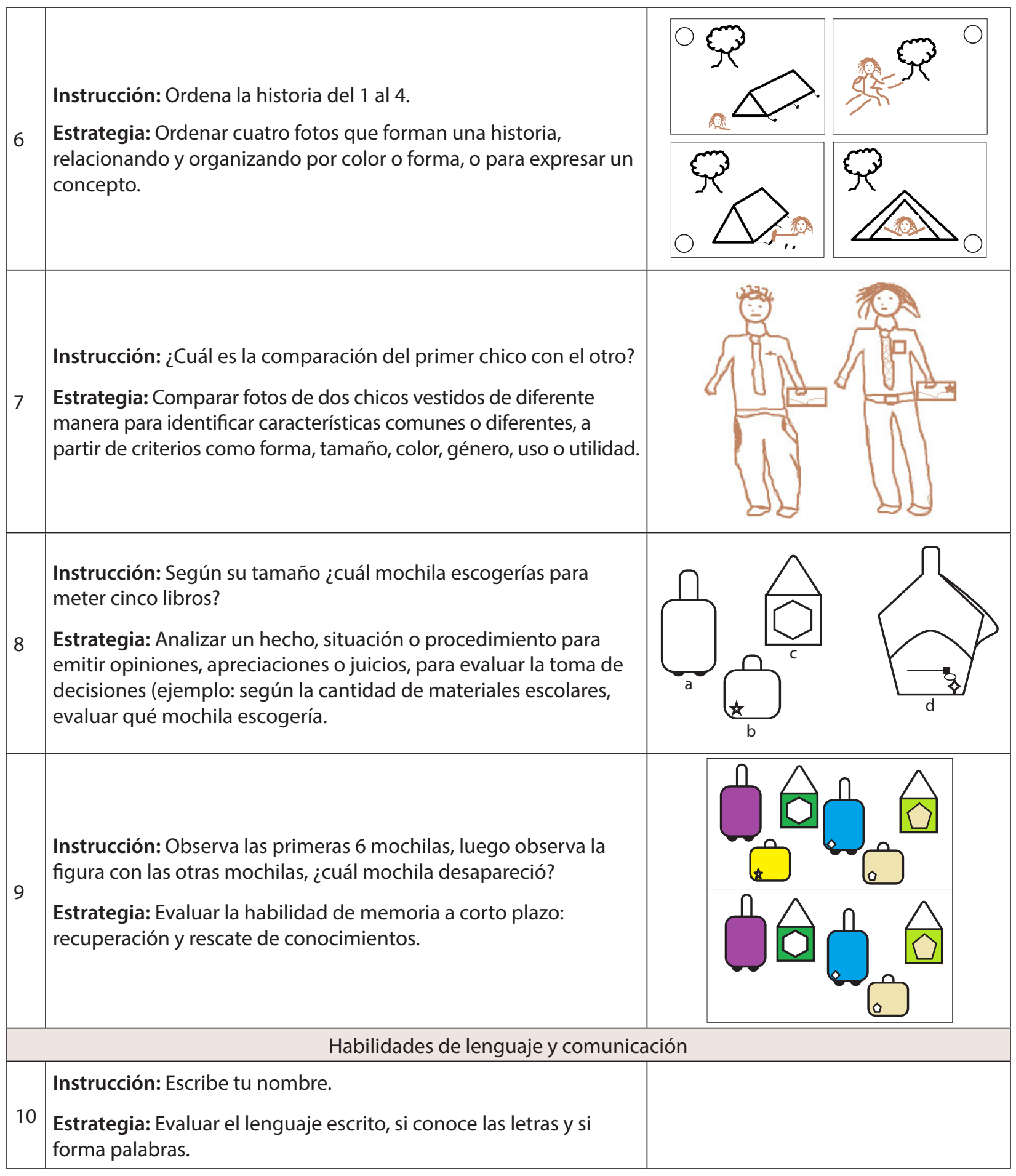

continúa 


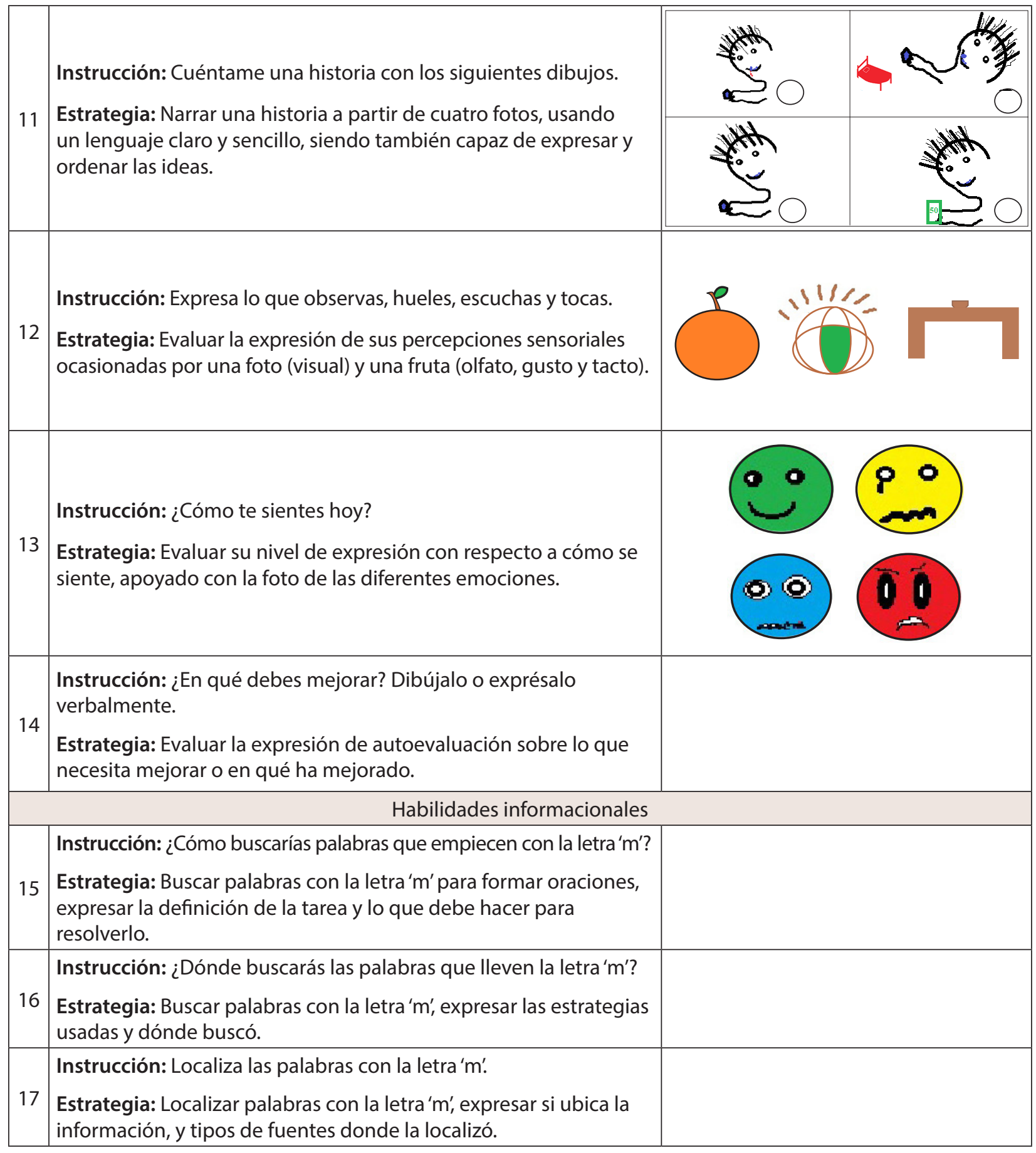

continúa 
http://doi.org/10.15359/ree.24-3.21

http://www.una.ac.cr/educare

educare@una.ac.cr

\begin{tabular}{|l|l|l|}
\hline 18 & Instrucción: Usa las palabras para formar oraciones. & \\
& $\begin{array}{l}\text { Estrategia: Usar palabras para formar oraciones y expresar su } \\
\text { decisión sobre cuáles palabras usar. }\end{array}$ & \\
\hline 19 & $\begin{array}{l}\text { Instrucción: Comunica las palabras que encontraste } \\
\text { Estrategia: Según las palabras que encontró, sintetizar } \\
\text { información y comunicar el resultado (oral o escrito). }\end{array}$ & \\
\hline 20 & $\begin{array}{l}\text { Instrucción: ¿Qué aprendiste? } \\
\text { Estrategia: Evaluar lo aprendido en el ejercicio anterior. Expresar } \\
\text { cómo evaluó, usó fuentes de información e identificar autor, y } \\
\text { título de la fuente. }\end{array}$ & \\
\hline
\end{tabular}

Nota: Elaboración propia.

\section{Segundo instrumento: Rúbrica para evaluar el examen diagnóstico}

Para diseñar el segundo instrumento, se consideraron diferentes criterios de contenido y del panel experto participante en la validación de los instrumentos, así como de un asesor externo, quienes evaluaron cada habilidad. La escala de evaluación del instrumento incluye los valores de: $(R=$ Regular), si se necesita enseñar la habilidad al niño o niña; $(B=B i e n)$; si practica la habilidad; $y$ ( $M B=M u y$ Bien), si domina la habilidad, donde, si obtiene $B$ o MB, estos resultados son aceptables. La Tabla 2 describe este segundo instrumento; presenta los tipos de habilidades, las habilidades que se evaluaron por cada tipo, además de los aspectos que se consideraron para evaluar cada una, utilizando la escala mencionada.

\section{Tercer instrumento: Tabla de resultados}

El tercer instrumento consiste en una tabla de resultados (ver Tabla 3). Una vez realizada la revisión del examen diagnóstico, se completó esta tabla de resultados, por cada participante en el estudio y de acuerdo con sus habilidades básicas de pensamiento, pensamiento matemático, lenguaje, comunicación e informacionales, empleando la mencionada escala de evaluación de $\mathrm{R}, \mathrm{B}$ y $\mathrm{MB}$.

Los resultados conjuntados producto de la rúbrica para evaluar el examen diagnóstico son integrados en el instrumento 3, el cual permitió dar cuenta de aquellas habilidades que en mayor o menor grado tenían desarrolladas el estudiantado de primer grado, lo cual permitió tomar decisiones sobre nuevas estrategias para un aprendizaje que favoreciera el desarrollo integral de la totalidad de los miembros del grupo participante. 
http://doi.org/10.15359/ree.24-3.21

http://www.una.ac.cr/educare educare@una.ac.cr

Tabla 2: Rúbrica para evaluar el examen diagnóstico

\begin{tabular}{|c|c|c|c|c|}
\hline \multicolumn{2}{|c|}{ Habilidades } & \multirow{2}{*}{\begin{tabular}{l}
\multicolumn{1}{c}{ Regular (R) } \\
Dificultad para poner \\
atención y dijo menos de \\
cuatro características.
\end{tabular}} & \multirow{2}{*}{$\begin{array}{l}\text { Bien (B) } \\
\begin{array}{l}\text { Identificó cuatro } \\
\text { características }\end{array}\end{array}$} & \multirow{2}{*}{$\begin{array}{l}\text { Muy bien (MB) } \\
\text { Puso atención, identificó el color, forma } \\
\text { y tamaño. Los elementos de clasificación } \\
\text { fueron: número, especie, uso, utilidad, } \\
\text { posición, orden y cantidad. }\end{array}$} \\
\hline $\begin{array}{l}\text { Básicas del } \\
\text { pensamiento } \\
\text { y pensamiento } \\
\text { matemático }\end{array}$ & 1. Observación & & & \\
\hline & 2. Clasificación & $\begin{array}{l}\text { Puso atención, dijo una } \\
\text { característica o no pudo } \\
\text { realizar la actividad. }\end{array}$ & $\begin{array}{l}\text { Puso atención } \\
\text { y dijo cuatro } \\
\text { características. }\end{array}$ & $\begin{array}{l}\text { Puso atención, identificó: semejanzas y } \\
\text { diferencias; categorías (clases y subclases) } \\
\text { de objetos, animales y cosas }\end{array}$ \\
\hline & 3. Interpretación & $\begin{array}{l}\text { Puso atención, dijo una } \\
\text { característica o no pudo } \\
\text { realizar la actividad. }\end{array}$ & $\begin{array}{l}\text { Identificó cuatro } \\
\text { características. }\end{array}$ & $\begin{array}{l}\text { Puso atención y dio orden a: imágenes, } \\
\text { datos, eventos; identificó situaciones y } \\
\text { eventos en un orden lógico. }\end{array}$ \\
\hline & 4. Representación & $\begin{array}{l}\text { Puso atención, dijo una } \\
\text { característica o no pudo } \\
\text { realizar la actividad. }\end{array}$ & $\begin{array}{l}\text { Identificó cuatro } \\
\text { características. }\end{array}$ & $\begin{array}{l}\text { Puso atención, dijo figura, objeto, } \\
\text { situación, significado, una idea, } \\
\text { condiciones, circunstancias y partes. }\end{array}$ \\
\hline & 5. Inferencias & $\begin{array}{l}\text { Dificultad para } \\
\text { concentrarse y resolver } \\
\text { el caso. }\end{array}$ & $\begin{array}{l}\text { Puso atención } \\
\text { y resolvió el } \\
\text { problema. }\end{array}$ & $\begin{array}{l}\text { Se concentró, resolvió el problema, dijo } \\
\text { consecuencias, reglas o principios generales } \\
\text { de la situación o caso concreto. }\end{array}$ \\
\hline & 6. Ordenación & Ordenó solo por forma. & $\begin{array}{l}\text { Ordenó por color, } \\
\text { género, forma. }\end{array}$ & $\begin{array}{l}\text { Ordenó, relacionó y organizó información } \\
\text { para expresar un concepto; organizó } \\
\text { elementos, sea por color o forma. }\end{array}$ \\
\hline & 7. Comparación & Dificultad para comparar. & $\begin{array}{l}\text { Identificó cuatro } \\
\text { características }\end{array}$ & $\begin{array}{l}\text { Puso atención, observó sistemáticamente, } \\
\text { comparó por género, figura, situación y } \\
\text { color. }\end{array}$ \\
\hline & $\begin{array}{l}\text { 8. Toma de } \\
\text { decisiones }\end{array}$ & $\begin{array}{l}\text { Dificultad para evaluar } \\
\text { y tomar una decisión } \\
\text { certera. }\end{array}$ & $\begin{array}{l}\text { Analizó y buscó } \\
\text { relaciones causa- } \\
\text { efecto. }\end{array}$ & $\begin{array}{l}\text { Observó y analizó el hecho, emitió opiniones, } \\
\text { apreciaciones o juicios, buscó relaciones } \\
\text { causa-efecto, tomó decisiones certeras. }\end{array}$ \\
\hline & 9. Memoria & Dificultad para recordar. & $\begin{array}{l}\text { Rescató algunos } \\
\text { elementos. }\end{array}$ & $\begin{array}{l}\text { Recuperó y rescató todos los elementos } \\
\text { importantes. }\end{array}$ \\
\hline \multirow[t]{5}{*}{$\begin{array}{l}\text { Lenguaje y } \\
\text { comunicación }\end{array}$} & $\begin{array}{l}\text { 10. Escribe su } \\
\text { nombre }\end{array}$ & $\begin{array}{l}\text { Faltaron letras en el } \\
\text { nombre. }\end{array}$ & $\begin{array}{l}\text { Nombre sin } \\
\text { apellidos. }\end{array}$ & Escribió su nombre completo. \\
\hline & $\begin{array}{l}\text { 11. Narra una } \\
\text { historia }\end{array}$ & $\begin{array}{l}\text { Se le complicó contar la } \\
\text { historia en secuencia. }\end{array}$ & $\begin{array}{l}\text { Contó historia en } \\
\text { orden. }\end{array}$ & $\begin{array}{l}\text { Contó historias por orden de ideas, fue } \\
\text { imaginativo y tuvo un vocabulario variado. }\end{array}$ \\
\hline & 12. Percepción & $\begin{array}{l}\text { Identificó características } \\
\text { por medio de un sentido } \\
\text { o no pudo realizar la } \\
\text { actividad. }\end{array}$ & $\begin{array}{l}\text { Identificó } \\
\text { características } \\
\text { por medio de dos } \\
\text { sentidos. }\end{array}$ & $\begin{array}{l}\text { Identificó características de los objetos } \\
\text { por medio de los sentidos vista, olfato, } \\
\text { gusto y tacto. }\end{array}$ \\
\hline & $\begin{array}{l}\text { 13. Comunica sus } \\
\text { emociones }\end{array}$ & $\begin{array}{l}\text { Dificultad para comunicarse } \\
\text { con otras personas. }\end{array}$ & $\begin{array}{l}\text { Habló de sus } \\
\text { emociones. }\end{array}$ & $\begin{array}{l}\text { Se desenvolvió oralmente y compartió sus } \\
\text { emociones. }\end{array}$ \\
\hline & 14. Autoevaluación & Dificultad para expresarse. & $\begin{array}{l}\text { Al menos dijo una } \\
\text { cosa que necesita. }\end{array}$ & $\begin{array}{l}\text { Se autovalora y conoce su capacidad para } \\
\text { hacer las cosas. }\end{array}$ \\
\hline
\end{tabular}


http://doi.org/10.15359/ree.24-3.21

http://www.una.ac.cr/educare

educare@una.ac.cr

\begin{tabular}{|c|c|c|c|c|}
\hline \multicolumn{2}{|c|}{ Habilidades } & \multirow{2}{*}{$\begin{array}{l}\text { Regular }(\mathrm{R}) \\
\text { Dificultad para identificar } \\
\text { el problema. }\end{array}$} & \multirow{2}{*}{$\begin{array}{l}\text { Bien (B) } \\
\text { Enfocó el } \\
\text { problema. }\end{array}$} & \multirow{2}{*}{$\begin{array}{l}\text { Muy bien (MB) } \\
\text { Define el problema. }\end{array}$} \\
\hline Informacionales & 15. Define tareas & & & \\
\hline & $\begin{array}{l}\text { 16. Búsqueda de } \\
\text { información }\end{array}$ & $\begin{array}{l}\text { Dificultad para buscar la } \\
\text { información. }\end{array}$ & $\begin{array}{l}\text { Supo cómo buscar } \\
\text { información. }\end{array}$ & $\begin{array}{l}\text { Describe la forma cómo realiza la } \\
\text { búsqueda y define su forma de búsqueda }\end{array}$ \\
\hline & $\begin{array}{l}\text { 17. Localización } \\
\text { y acceso a la } \\
\text { información }\end{array}$ & $\begin{array}{l}\text { Dificultad para localizar } \\
\text { información. }\end{array}$ & $\begin{array}{l}\text { Obtuvo } \\
\text { información. }\end{array}$ & $\begin{array}{l}\text { Reconoce lo que encuentra e identifica lo } \\
\text { que obtuvo de información }\end{array}$ \\
\hline & $\begin{array}{l}\text { 18. Análisis de } \\
\text { información }\end{array}$ & $\begin{array}{l}\text { Dificultad para extraer } \\
\text { información relevante. }\end{array}$ & $\begin{array}{l}\text { Leyó, escuchó y } \\
\text { vio la información. }\end{array}$ & $\begin{array}{l}\text { Extrae la información relevante de la } \\
\text { fuente. }\end{array}$ \\
\hline & $\begin{array}{l}\text { 19. Sintetiza } \\
\text { información }\end{array}$ & $\begin{array}{l}\text { Dificultad para organizar la } \\
\text { información. }\end{array}$ & $\begin{array}{l}\text { Organizó la } \\
\text { información. }\end{array}$ & $\begin{array}{l}\text { Organiza la información y sabe a quién va } \\
\text { dirigida. }\end{array}$ \\
\hline & $\begin{array}{l}\text { 20. Evaluación de } \\
\text { la información }\end{array}$ & Dificultad para evaluar. & $\begin{array}{l}\text { Identificó lo que } \\
\text { aprendió. }\end{array}$ & $\begin{array}{l}\text { Identificó lo que aprendió y para qué le } \\
\text { va a servir. } \\
\text { Reflexiona sobre la información. }\end{array}$ \\
\hline
\end{tabular}

Nota: Elaboración propia.

Tabla 3: Tabla de resultados

\begin{tabular}{|c|c|c|c|c|c|c|c|c|c|c|c|c|c|c|c|c|c|c|c|c|}
\hline & \multicolumn{10}{|c|}{$\begin{array}{l}\text { Habilidades básicas de pensamiento y } \\
\text { pensamiento matemático }\end{array}$} & \multicolumn{4}{|c|}{$\begin{array}{l}\text { Lenguaje y } \\
\text { comunicación }\end{array}$} & \multicolumn{6}{|c|}{ Habilidades informacionales } \\
\hline 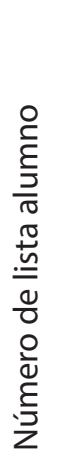 & 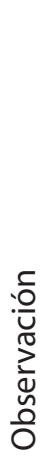 & 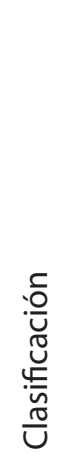 & 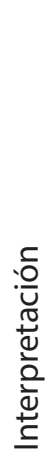 & 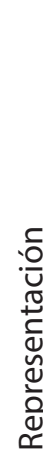 & 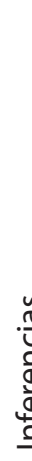 & & 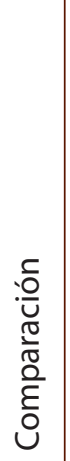 & 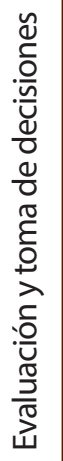 & 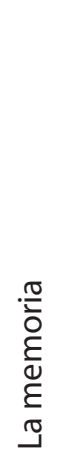 & 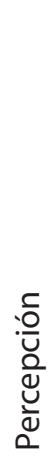 & 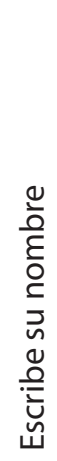 & 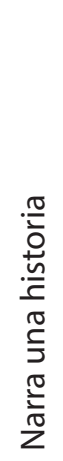 & 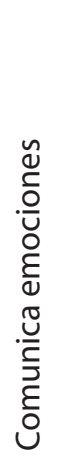 & 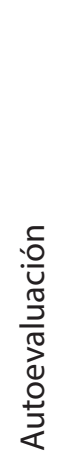 & $\begin{array}{l}\frac{0}{\varepsilon} \\
\frac{0}{0} \\
0 \\
\frac{0}{0} \\
\overline{0} \\
. \bar{E} \\
\frac{1}{0} \\
0\end{array}$ & 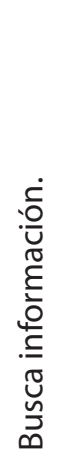 & 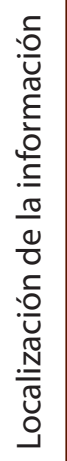 & $\frac{\frac{n}{\frac{n}{n}}}{\frac{\pi}{\pi}}$ & 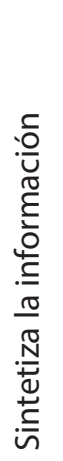 & 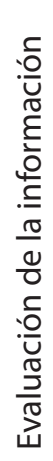 \\
\hline 1 & & & & & & & & & & & & & & & & & & & & \\
\hline 2 & & & & & & & & & & & & & & & & & & & & \\
\hline 3 & & & & & & & & & & & & & & & & & & & & \\
\hline 4 & & & & & & & & & & & & & & & & & & & & \\
\hline 5 & & & & & & & & & & & & & & & & & & & & \\
\hline$\ldots$ & & & & & & & & & & & & & & & & & & & & \\
\hline
\end{tabular}

Nota: Elaboración propia. 
http://doi.org/10.15359/ree.24-3.21

\section{Resultados}

El diseño y aplicación de los tres instrumentos antes descritos, en el caso de este estudio, en su parte experimental, permitió al personal docente identificar las habilidades que poseía el estudiantado de primer grado de educación básica. La aplicación de estos instrumentos permitió obtener resultados concretos de las condiciones de los sujetos participantes y, a partir de ello, reorientar la futura planeación pedagógica situacional y así buscar un mayor éxito en la enseñanza de la lectoescritura.

De acuerdo con los resultados obtenidos, fue posible observar que, para 27 estudiantes participantes (14 niños y 13 niñas), conforme a las 20 habilidades evaluadas, el comportamiento fue el siguiente (ver Figura 1):

a) La habilidad que sobresalió con una calificación de regular fue la de observación, ya que la mayoría señaló de tres a cuatro características, incluyendo color, forma y cantidad.

b) De la misma forma, al evaluar de manera grupal, las habilidades sobresalientes fueron: autoevaluación, retención, percepción, interpretación, representación, ordenación, retención y escribir su nombre (en la mayoría sin apellidos).

c) La media general del grupo sobre la cantidad de habilidades en las que demostraron competencia fue de 7.148, en tanto las niñas obtuvieron una media superior de 7.46 y en los niños, la media fue inferior a las dos anteriores, correspondiendo a 6.38 habilidades en promedio.

d) Solo tres estudiantes (entre ellos dos niñas y un niño) ofrecieron resultados distintivos al demostrar competencia de 11 a 14 habilidades de las 20 evaluadas, que incluyeron la observación, clasificación, interpretación, representación, inferencias, ordenación, comparación, evaluación y toma de decisiones, memoria, escribir su nombre, narra la historia, percepción, comunica sus emociones y mencionaron en su autoevaluación que podían mejorar en la lectura y escritura.

e) Se observó, además, que tres estudiantes que sobresalieron en la evaluación de habilidades se encontraban en la etapa silábica-alfabética de la escritura, mientras que los demás no, encontrando el hallazgo que, las dos niñas y un niño demostraron un mejor nivel de desarrollo de sus habilidades, también fue notoria su habilidad de lecto-escritura.

f) Respecto a las habilidades informacionales, se observaron resultados de baja presencia y desarrollo, en los cuales fue posible reconocer que todo el grupo de participantes en el estudio demandaban apoyo pedagógico para el desarrollo de estas habilidades.

g) De 27 estudiantes, según los resultados individuales, 24 requieren el desarrollo de habilidades para la lectura, escritura, la observación, clasificación, interpretación, representación, inferencias, ordenación, comparación, evaluación y toma de decisiones, memoria, escribir su nombre, narrar las historias, percepción, comunicar sus emociones. 
http://doi.org/10.15359/ree.24-3.21

http://www.una.ac.cr/educare

educare@una.ac.cr

h) Preocupa, de manera importante, que dos participantes en el estudio solo demostraran capacidad en dos habilidades y dos estudiantes más en cuatro habilidades, lo cual demandará una atención directa y especial sobre sus procesos formativos. Esta situación es preocupante, dado que el personal docente no posee las condiciones de infraestructura y de formas de trabajo para centrase solo en estos casos sin descuidar las condiciones generales del grupo.

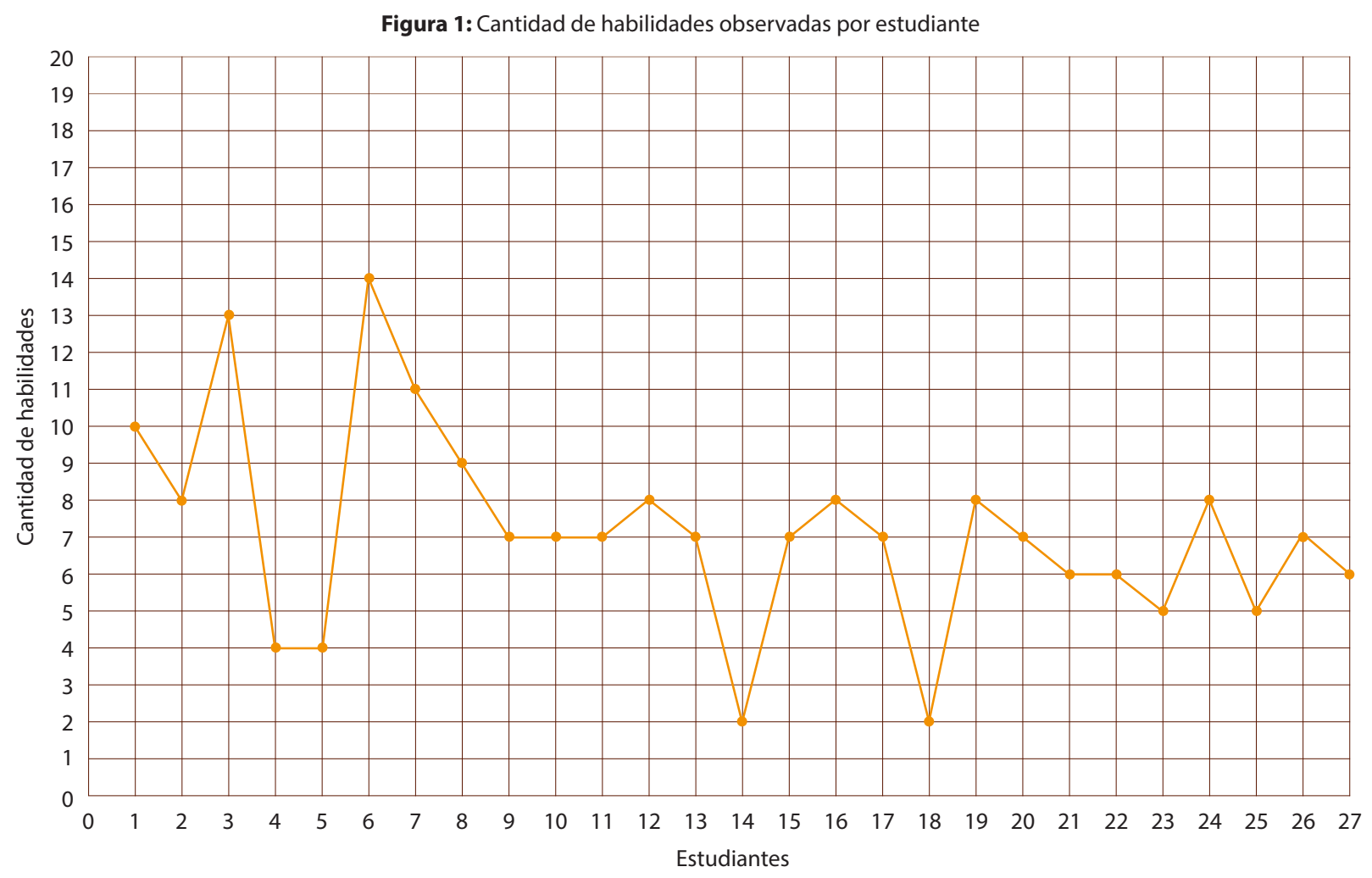

Nota: Elaboración propia.

Cabe destacar que, si el estudiantado obtiene resultados de 11 a más habilidades en el diagnóstico, existen mayores probabilidades de que obtenga mejores resultados académicos en el primer grado de primaria, es decir, que demuestre su capacidad de lectoescritura. Aunque el número de actividades es bajo ( 11 de 20) para considerar la suficiencia en estas habilidades, de cualquier forma, el personal docente deberá desarrollar estrategias que fortalezcan la competencia de lectoescritura, haciendo especial hincapié en el fortalecimiento de las habilidades y la inclusión de estas en los contenidos curriculares, de tal forma que se lleven a la práctica en las orientaciones pedagógicas del grupo. 
http://doi.org/10.15359/ree.24-3.21

http://www.una.ac.cr/educare educare@una.ac.cr

El análisis previo representa el análisis de habilidades demostradas con resultados suficientes por cada estudiante del grupo, sin embargo, resulta pertinente estudiar el comportamiento de cada habilidad a nivel grupal. Por medio de la Figura 2 es posible observar el comportamiento grupal por actividad, de lo cual se derivan los siguientes hallazgos:

a) En cuanto a las seis habilidades informacionales evaluadas, todo el grupo de participantes obtuvo resultados de valoración de R, lo cual se considera crítico, ya que estas habilidades deben ser aprendizas desde primer grado para lograr un aprendizaje permanente.

b) Las habilidades que más se aprecian con una escala de MB y B son: autoevaluación, comunicar sus emociones, comparación, evaluación y toma de decisiones, ordenación, inferencias y la observación.

c) Los resultados que obtuvieron algunos niños y niñas en la escala de B, lo cual se considera como aceptable en la evaluación, se centran en las siguientes habilidades: percepción, interpretación, representación, retención, escribir su nombre, autoevaluación, inferencia, ordenación y toma de decisiones.

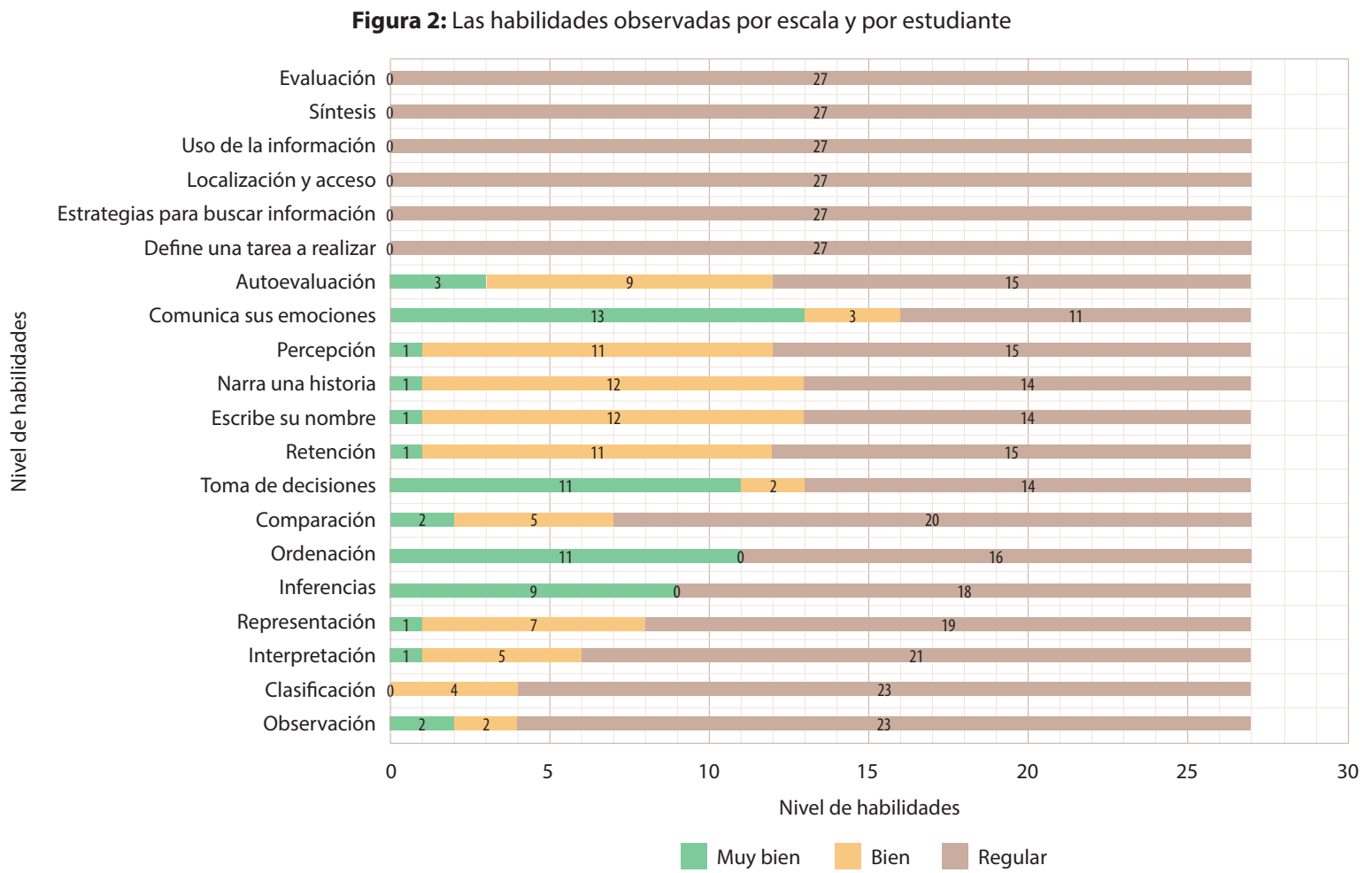

Nota: Elaboración propia. 
http://doi.org/10.15359/ree.24-3.21

http://www.una.ac.cr/educare

educare@una.ac.cr

Los resultados anteriores muestran alta variabilidad en el comportamiento de cada una de las habilidades. La mayoría de estudiantes se encuentra en condición de $\mathrm{R}$, lo cual representa un reto para el personal docente al pretender lograr que lleguen a otra condición. En cuanto a las habilidades con presencia en la escala de evaluación de $\mathrm{B}$ y $\mathrm{MB}$, estas condiciones mayormente facilitarán al personal docente sus procesos de enseñanza, es decir, el tiempo que se dedica será menor para lograr el aprendizaje.

A mayor número de habilidades que resulten en la escala de evaluación con calificación de $\mathrm{R}$, será mayor la necesidad de generar diversas estrategias específicas para su desarrollo, lo cual significa que el estudiantado requerirá de mayor tiempo y esfuerzo para integrar las habilidades de lectoescritura y otros contenidos en sus procesos formativos. De tal modo, que las estrategias didácticas que se planeen deberán llevar consigo elementos que desarrollen habilidades de pensamiento, lingüísticas e informacionales.

Con el fin de objetivar los resultados, se procedió a su análisis basado en porcentajes, diferenciando entre niños y niñas (ver Figura 3). Dado que los resultados por habilidad, en general se centran en la categoría de $\mathrm{R}$, existe una tendencia a mostrar porcentajes bajos. Para este caso, solo tres estudiantes obtuvieron arriba del $50 \%$ (52 \% una niña, $62 \%$ un niño y $67 \%$ una niña), el resto de los resultados observan como máximo un 43 \% y como mínimo un $10 \%$.

\section{Discusión y conclusiones}

Resulta complejo atribuir las condiciones de aprendizaje de niños y niñas entre seis y siete años al papel que juega el personal docente dentro del salón de clase. En el ambiente infantil se presentan múltiples factores que determinan los comportamientos personales y académicos, por tanto, un DS está determinado por factores fundamentales como la salud física y emocional del estudiantado, por ejemplo, es crucial, para el futuro de las sociedades, que la niñez pueda alcanzar un desarrollo físico y psicológico óptimo. Son importantes la nutrición y salud, los cuidados afectuosos y el estímulo para aprender en los primeros años de vida, ya que ayuda a obtener mejores resultados académicos, estar más saludables, fuertes y participar en la sociedad. En tales factores, en la mayoría de los casos, el personal docente tiene poca posibilidad de influir.

Para el caso de este estudio, se trata de influir en las habilidades de lectoescritura, como un elemento fundamental en el proceso de enseñanza y aprendizaje en etapa preoperacional, lo cual comprende, además, el desarrollo de diversas habilidades de pensamiento, pensamiento matemático, informacionales, de lenguaje y comunicación. Por ejemplo, el desarrollo de habilidades de pensamiento en particular resulta crucial, ya que es la parte fundamental para aprender a leer y a escribir, esto representa el alto vínculo entre la alfabetización lectora con el desarrollo de habilidades informacionales. 
Figura 3: Porcentajes obtenidos por estudiante (niños y niñas)

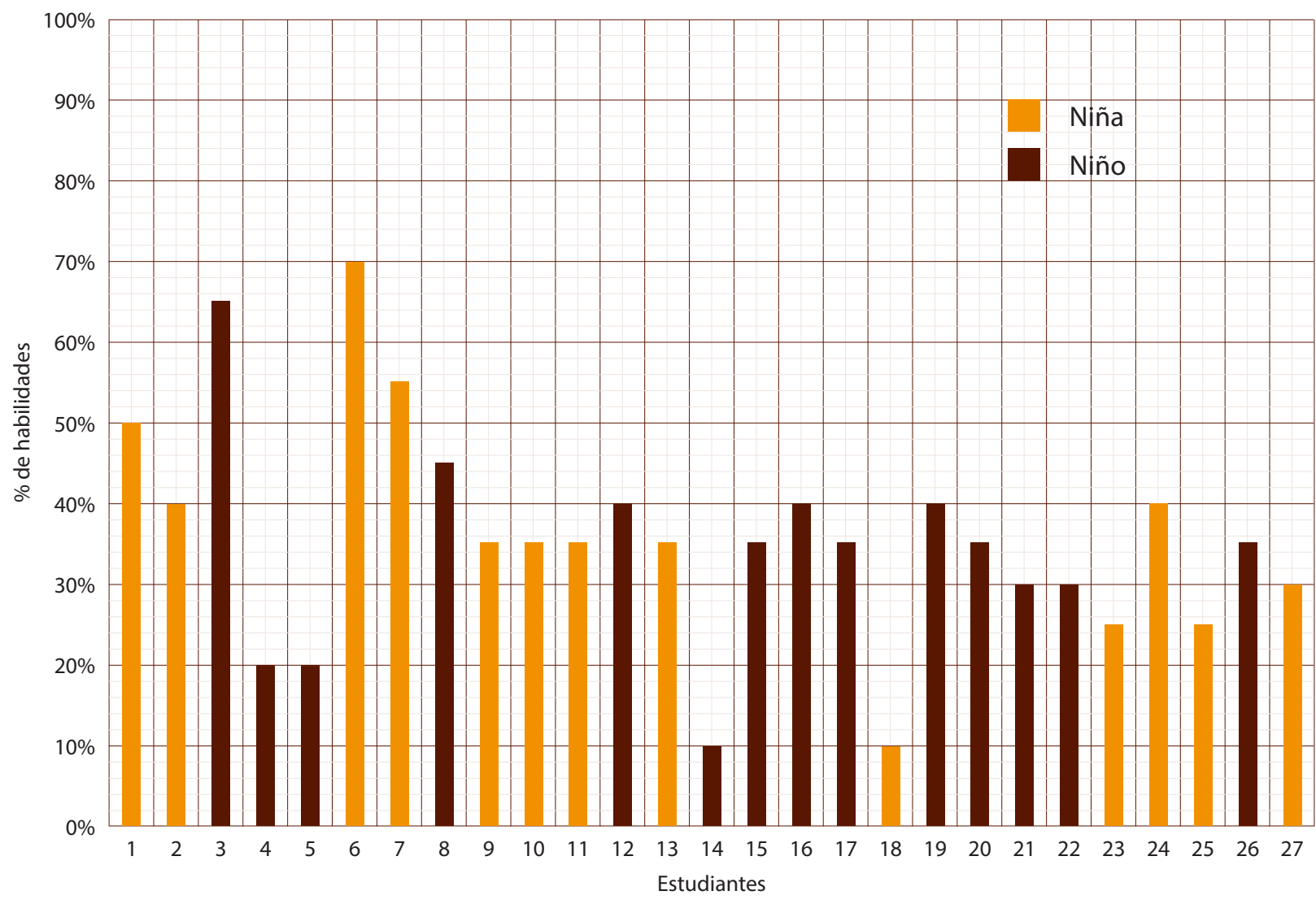

Nota: Elaboración propia.

Se sabe que para leer se necesita de las habilidades de pensamiento como la observación, identificación, ubicación, interpretación, atención, la percepción, así como de la identificación de códigos mediante la memoria y procesos cognitivos. Luego, la escritura necesita de las habilidades motrices, habilidades cognitivas, la observación, identificación, percepción y la memoria. Finalmente, el desarrollo de habilidades informacionales permite apoyar todas las demás habilidades.

Las habilidades informacionales como tales, en todos los niveles educativos, resultan fundamentales, ya que permiten a los grupos de estudiantes aprender a ubicar y comunicar la información. Además, resultan esenciales, considerando que en países como México existen poblaciones amplias cursando la educación primaria. Esto significa que, si tales habilidades no son desarrolladas en edad temprana, el estudiantado en niveles académicos posteriores, observará incapacidad de solucionar problemas, y se demandará, con ello, la urgente necesidad 
http://doi.org/10.15359/ree.24-3.21

http://www.una.ac.cr/educare

educare@una.ac.cr

de integrar, en la formación de estudiantes entre seis y siete años, las estrategias didácticas para la metacognición.

Según los resultados de esta investigación, el estudiantado entre seis y siete años de edad, al inicio del ciclo escolar de primer grado de educación básica, demostró cierto desarrollo de habilidades básicas de pensamiento, tales como: observación, comparación, clasificación, ordenación, inferencias, comunicación de emociones y solo escribió su nombre sin apellidos, lo cual da pauta a pensar que está preparado para aprender la lectoescritura, sin embargo, dentro de ese proceso es importante enfatizar en las orientaciones pedagógicas cada una de las habilidades planteadas en el DS, precisamente en las habilidades informacionales, en donde los resultados merecen mayor atención.

Se reitera la importancia de las habilidades informacionales, ya que se concluye que los niños y niñas de primer grado no tienen la noción del concepto de información, por lo que resulta complicado evidenciar su nivel de desarrollo de tales habilidades. Dada esta condición, la retrospección comienza en el año escolar, cuando el estudiantado, en su consciencia reflexiva comienza a repensar conceptos y el lenguaje se vuelve fundamental cuando este se desarrolla con la enseñanza del concepto. Esto se infiere por su edad, ya que está en la etapa preoperatoria y no ha tenido experiencias conscientes con estas habilidades. Aunque los resultados obtenidos parecieran bajos, es importante considerar que en esta edad se tiene preparación para desarrollar la habilidad de lectoescritura.

El diagnóstico en sí ofrece resultados concretos; sin embargo, el éxito del estudiantado depende también de los cuidados que tuvo antes de entrar a la etapa escolar y después de esta misma. Los resultados del diagnóstico indican que en la edad de seis y siete años se tienen desarrolladas pocas habilidades de pensamiento, y sobresalen la observación, comparación, clasificación, ordenación, inferencias, comunicación de emociones, memoria y narración de historias con un lenguaje corto y no estructurado, y no manifestó ninguna habilidad informacional.

Es pertinente indicar que, mientras no tengan la experiencia de desarrollar habilidades informacionales, esta población infantil no demostrará un incremento de estas en ningún diagnóstico. Mientras no se le enseñe o se le haga saber al estudiantado sobre las habilidades de pensamiento, informacionales y lingüísticas que debe desarrollar, se le hará más fácil aprenderlas conscientemente.

Los resultados muestran la oportunidad de plantear estrategias dirigidas a situaciones didácticas que permitan el desarrollo de habilidades básicas de pensamiento, de pensamiento matemático, lingüísticas e informacionales. Se recomienda realizar una planeación para la organización docente, que puede hacerse por día, semana o mes, con evaluación y retroalimentación y estar estructurada. Se concluye que los niños y las niñas con más avance en 
http://doi.org/10.15359/ree.24-3.21

la lectoescritura sobresalen más en sus habilidades en el DS, es decir, quienes están en la etapa silábica-alfabética y quienes están en la silábica observaron menos habilidades evaluadas. El DS resulta fundamental para la mejora continua, tanto de la docencia como la calidad en la educación.

Se recomienda, para la enseñanza de las habilidades informacionales, la implantación de cinco fases: definir necesidades de información, buscar información, localizar la información, analizar y comunicar la información. Se recomienda que todo el cuerpo docente que imparta clases de primer grado de primaria parta de la realización de un DS y que tenga como prioridad enseñar habilidades de pensamiento e informacionales con la transversalidad de la lectoescritura con acciones tales como: explicar una palabra, dar a conocer el concepto, identificar relaciones y permitir cuestionamientos sobre todo donde se podría encontrar la información. Con ello, se llegará a la formación de alumnado crítico, analítico y creativo durante su proceso de lectoescritura, como parte de la formación presente y futura. Se concluye que diagnosticar las condiciones de DS es fundamental para así visualizar, desde el comienzo de ciclo escolar, las áreas de oportunidad en la niñez.

\section{Agradecimientos}

Proyecto financiado por el Consejo Nacional de Ciencia y Tecnología (CONACYT) de México, a través del Programa Nacional de Posgrados de Calidad (PNPC), con apoyo de Beca Nacional para estudios de posgrado en la Universidad Autónoma de Chihuahua (México).

\section{Declaración de Material complementario}

Este artículo tiene disponible como material complementario:

- La versión preprint del artículo en https://doi.org/10.5281/zenodo.3364193

\section{Referencias}

Amestoy de Sánchez, M. (2002). La investigación sobre el desarrollo y la enseñanza de las habilidades de pensamiento. Revista Electrónica de Investigación Educativa, 4(1), 1-32. https://redie.uabc.mx/redie/article/view/55

Araya, N. (2014). Las habilidades de pensamiento y el aprendizaje significativo en matemática, de escolares de quinto grado en Costa Rica. Actualidades Investigativas en Educación, 14(2), 1-30. https://doi.org/10.15517/aie.v14i2.14744

Arriaga Hernández, M. (2015). El diagnóstico educativo, una importante herramienta para elevar la calidad de la educación en manos de los docentes. Atenas, 3(31), 63-74. https://www. redalyc.org/pdf/4780/478047207007.pdf 
http://doi.org/10.15359/ree.24-3.21

http://www.una.ac.cr/educare

educare@una.ac.cr

Bahmani, S. (2016). Improved critical thinking in students using current events journaling. International Journal of Sociology and Social Policy, 36(3-4), 190-202. https://doi. org/10.1108/ijssp-04-2015-0038

Barboza Marcano, Y. (2007). La lectura: Herramienta fundamental en la formación de los futuros docentes en el contexto de la sociedad del conocimiento. Laurus, 13(24), 112-130. https:// www.redalyc.org/pdf/761/76111485006.pdf

Beck, D. (2015). The online school librarian: Roles and responsibilities. TechTrends, 59(3), 77-84. https://doi.org/10.1007/s11528-015-0856-8

Blummer, B. y Kenton, J. M. (2014). Information problem solving and metacognitive skills. En Improving student information search: A metacognitive approach (pp. 45-54). Chandos Publishing. https://doi.org/10.1533/9781780634623.45

Brier, D. J. y Lebbin, V. K. (2015). Learning information literacy through drawing. Reference Services Review, 43(1), 45-67. https://doi.org/10.1108/rsr-08-2014-0030

Costa, C., Tyner, K., Henriques, S., y Sousa, C. (2018). Game creation in youth media and information literacy education. International Journal of Game-Based Learning, 8(2), 1-13. https://doi.org/10.4018/ijgbl.2018040101

Drotner, K., y Kobbernagel C. (2014). Toppling hierarchies? Media and information literacies, ethnicity, and performative media practices. Learning, Media and Technology, 39(4), 409428. https://doi.org/10.1080/17439884.2014.964255

Durand, M., Hulme, C., Larkin, R. y Snowling, M. (2005). The cognitive foundations of reading and arithmetic skills in 7- to 10-years-olds. Journal of Experimental Child Psychology, 91(2), 113136. https://doi.org/10.1016/j.jecp.2005.01.003

Foo, S., Majid, S., Azura Mokhtar, I., Zhang, X., Chang, Y.-K., Luyt, B.yTheng, Y.-L. (2014). Information literacy skills of secondary school students in Singapore. Aslib Journal of Information Management, 66(1), 54-76. https://doi.org/10.1108/AJIM-08-2012-0066

García Retana J. A. (2011). Modelo educativo basado en competencias: Importancia y necesidad. Revista Actualidades Investigativas en Educación, 11(3), 1-24. https://www.redalyc.org/ pdf/447/44722178014.pdf

González-López, M. (2019). La enseñanza de habilidades de pensamiento y de la comprensión lectora en estudiantes de bajo rendimiento escolar. Areté. Revista Digital del Doctorado en Educación de la Universidad Central de Venezuela, 5(9), 145-167. http://saber.ucv.ve/ojs/ index.php/rev arete/article/view/16284 
http://doi.org/10.15359/ree.24-3.21 http://www.una.ac.cr/educare educare@una.ac.cr

González-López, M.,Machin-Mastromatteo, J.D.yTarango,J. (2019). Alfabetización Informacional: Enseñanza y desarrollo de su competencia en la educación básica. E-Ciencias de la Información, 9(2), 1-19. https://doi.org/10.15517/eci.v9i2.35774

González-López, M. (2020). Habilidades para desarrollar la lectoescritura en niños de educación primaria. Revista Estudios en Educación, 3(4), 45-68. http://ojs.umc.cl/index.php/ estudioseneducacion/article/view/83/63

Heichman, L. (2008). Information literacy in subject specific vocabularies: A path to critical thinking. College \& Undergraduate Libraries. 15(1-2), 141-158. https://doi. org/10.1080/10691310802177127

Heider, K. L. y Jalongo, M. R. (2015). Young children and families in the information age:Applications of technology in early childhood. Springer. https://doi.org/10.1007/978-94-017-9184-7

Hidalgo, N. y Murillo, F.J. (2017). Las concepciones sobre el proceso de evaluación del aprendizaje de los estudiantes. REICE. Revista Iberoamericana sobre Calidad, Eficacia y Cambio en Educación, 15(1), 107-128. https://doi.org/10.15366/reice2017.15.1.007

Joo, K. H. y Park, N. H. (2015). e-PBL model development for computer learning system. International Journal of Multimedia and Ubiquitous Engineering, 10(3), 323-332. https://doi. org/10.14257/ijmue.2015.10.3.29

Lau, J., Machin-Mastromatteo, J. D., Gárate, A. y Tagliapietra-Ovies, A. C. (2016). Assessing Spanish-speaking university students' info-competencies with iSkills, SAILS, and an inhouse instrument: Challenges and benefits. Communications in Computer and Information Science, 676, 327-336. https://doi.org/10.1007/978-3-319-52162-6 32

Lovatón Sarco, P. F. (2012). Diagnóstico situacional: Herramienta indispensable en la elaboración del currículo para la formación académico profesional del comunicador social, al 2011 [Tesis de maestría]. Universidad Nacional Mayor de San Marcos, Perú. http://cybertesis.unmsm. edu.pe/handle/cybertesis/3074

Meléndez, S. y Gómez, L. J. (2008). La planificación curricular en el aula: Un modelo de enseñanza por competencias. Laurus, 14(26), 367-392. https://www.redalyc.org/ pdf/761/76111491018.pdf

Morales Vitela, C. T., Lau Noriega, J. y López Ornelas, M. (2018). Parámetros para el diseño de instrumentos en la evaluación del desarrollo de habilidades, competencias y alfabetización informacional. Revista Lumina, 12(1), 124-145. https://doi.org/10.34019/1981-4070.2018. $\underline{v 12.21490}$ 
http://doi.org/10.15359/ree.24-3.21

http://www.una.ac.cr/educare

educare@una.ac.cr

Morán Oviedo, P. (2004). La docencia como recreación y construcción del conocimiento. Sentido pedagógico de la investigación en el aula. Perfiles educativos, 26(105-106), 41-72. http:// www.scielo.org.mx/scielo.php?script=sci arttext\&pid=S0185-26982004000100003\&lng= es\&nrm=iso\&tlng=es

Moreno Olivos, T. (2012). La evaluación de competencias en educación. Sinéctica, 39, 1-20. http://www.scielo.org.mx/pdf/sine/n39/n39a10.pdf

Muria Vila, I. D. y Díaz Milagros, D. (2008). Desarrollo de las habilidades del pensamiento en los diferentes niveles educativos. Revista Electrónica de Psicología Iztacala, 11(1), 141-151. http://www.journals.unam.mx/index.php/repi/article/view/18549/17607

Navarro Navarro, V. (2018). Metodologías interdisciplinares como herramientas para motivar a alumnado de altas capacidades. Revista lberoamericana de Educación, 78(1), 43-66. https:// doi.org/10.35362/rie7813227

Organisation for Economic Co-operation and Development (2017a). PISA 2015 Assessment and analytical frame work: Science, reading, mathematic, financial literacy and collaborative problem solving [revised edition]. OCDE. https://doi.org/10.1787/9789264281820-en

Organisation for Economic Co-operation and Development (2017b). PISA 2015 assessment and analytical framework: Science, reading, mathematic, financial literacy and collaborative problem solving, [revised edition]. OCDE. https://doi.org/10.1787/9789264281820-en

Organización para la Cooperación y el Desarrollo Económico (2011). La medición del aprendizaje de los alumnos: Mejores prácticas para evaluar el valor agregado de las escuelas. OCDE. http://www.oecd.org/education/school/47871357.pdf

Paul, R. y Elder, L. (2003). La mini-guía para el pensamiento crítico: Conceptos y herramientas. Fundación para el Pensamiento Crítico. https://www.criticalthinking.org/resources/pdf/ sp-conceptsandtools.pdf

Pompa Montes de Oca, Y. de la C. y Pérez López, I. A. (2015). La competencia comunicativa en la labor pedagógica. Revista Universidad y Sociedad, 7(2), 160-167. https://rus.ucf.edu.cu/ index.php/rus/article/view/273

Ponjuan, G., Pinto, M. y Uribe-Tirado, A. (2015). Conceptualización y perspectivas de la alfabetización informacional en Iberoamérica: Un estudio Delphi. Information Research, 20(3), 1-29. http://eprints.rclis.org/28504/

Rodney-Wellington, K. (2014). An examination of information literacy instruction on the information seeking skills of primary school children in Jamaica: An experiment using grade six students. Journal of Information Literacy, 8(2), 73-100.https://doi.org/10.11645/8.2.1913 
http://doi.org/10.15359/ree.24-3.21

Rodríguez, R. (2008). ¿Cómo investigar con niños? En P. Martínez (Ed.), Cualitativa-mente: Los secretos de la investigación cualitativa (pp. 129-146). ESIC.

Secretaría de Educación Pública. (2017b). Aprendizajes clave para la educación integral: Plan y programasdeestudioparalaeducaciónbásica.Autor.https://www.planyprogramasdestudio. sep.gob.mx/descargables/APRENDIZAJES CLAVE PARA LA EDUCACION INTEGRAL.pdf

Secretaría de Educación Pública (2017). Aprendizajes clave para la educación integral. Tutoría y educación socioemocional. Educación secundaria. Plan y programas de estudio, orientaciones didácticas y sugerencias de evaluación. Autor. https://www. planyprogramasdestudio.sep.gob. $\mathrm{mx} /$ descargables/biblioteca/secundaria/tutoriasocioemocional/v1-programas-de-estudiop148-155.pdf

Siu Cheung, K. (2015). An experience of a three-year study on the development of critical thinking skills in flipped secondary classrooms with pedagogical and technological support. Computers \& Education, 89, 16-31. https://doi.org/10.1016/j.compedu.2015.08.017

Tamayo-Rueda, D., Moyares-Norchales, Y., Vigoa-Machin, L., Toll-Palma, Y del C., Falcon-Pi, G., Lemagne-Adán, A. y Rodríguez-González, L. (2012). Diagnóstico del grado de alfabetización informacional en los profesionales del centro de tecnologías para la formación de la universidad de las ciencias informáticas. Revista Española de Documentación Científica, 35(2), 347-360. https://doi.org/10.3989/redc.2012.2.856

Tapia, M. y Luna, J. (2010). Validación de una prueba de habilidades de pensamiento para alumnos de cuarto y quinto de secundaria y primer año de universidad. Revista de Investigación en Psicología, 13(2), 17-59. https://doi.org/10.15381/rinvp.v13i2.3710

Vilar, P. (1992). El diagnóstico de situación, una técnica para el análisis de alternativas y la valoración de sus consecuencias. Comunicación, Lenguaje y Educación, 4(15), 99-106. https://doi.org/10.1080/02147033.1992.10821038

Zavala, H. A. (2014). El desarrollo de las habilidades de pensamiento en el programa de filosofía para niños. Espiral:Revista de Docencia elnvestigación, 4(1),59-70.https://doi.org/10.15332/ erdi.v4i1.551

Zubac, A. y Tufekčić, A. (2014). Informacijska pismenost u svijetu i Hrvatskoj - rad školskoga knjižničara u osnovnoj školi. Vjesnik bibliotekara Hrvatske, 57(4), 221-238. https://hrcak. srce.hr/142320 